\title{
Analysis of rice RNA-dependent RNA polymerase 1 (OsRDR1) in virus-mediated RNA silencing after particle bombardment
}

\author{
Hui Chen · Atsushi Tamai - Masashi Mori - Masashi Ugaki · Yoshikazu Tanaka • \\ Partha P. Samadder • Akio Miyao - Hirohiko Hirochika • Naoto Yamaoka • \\ Masamichi Nishiguchi
}

Received: 4 November 2009/ Accepted: 5 January 2010/Published online: 23 February 2010

(C) The Phytopathological Society of Japan and Springer 2010

\begin{abstract}
RNA-dependent RNA polymerases (RDRs) play a key role in various RNA silencing pathways in many organisms. Using the nucleotide sequence of SGS2/SDE1/ RDR6 in Arabidopsis as the search query for sequences that flank the insertions of rice retrotransposon Tos 17 , we selected rice mutant lines $(O s R D R 1)$. RT-PCR analysis showed that OsRDRI mRNA was undetectable in leaves and calli of the mutants, while it was detected in wild type. RNA silencing
\end{abstract}

Electronic supplementary material The online version of this article (doi:10.1007/s10327-010-0226-5) contains supplementary material, which is available to authorized users.

H. Chen · N. Yamaoka · M. Nishiguchi $(\bowtie)$

Faculty of Agriculture, Ehime University,

3-5-7 Tarumi, Matsuyama, Ehime 790-8566, Japan

e-mail: mnishigu@agr.ehime-u.ac.jp

\section{A. Tamai · M. Mori}

Research Institute for Bioresources and Biotechnology,

Ishikawa Prefectural University, 1-308 Suematsu,

Nonoichi-machi, Ishikawa 921-8836, Japan

M. Ugaki

Department of Integrated Biosciences, The University of Tokyo,

5-1-5 Kashiwanoha, Kashiwa, Chiba 277-8562, Japan

Y. Tanaka $\cdot$ P. P. Samadder · A. Miyao $\cdot$ H. Hirochika

National Institute of Agrobiological Sciences,

2-1-2 Kan-nondai, Tsukuba, Ibaraki 305-8602, Japan

Present Address:

Y. Tanaka

Wakasa Wan Energy Research Center, Tsuruga,

Fukui 914-0192, Japan

Present Address:

P. P. Samadder

Department of Immunobiology, College of Medicine,

University of Arizona, Tucson, AZ 85724, USA was induced by particle bombardment to investigate any effects of $O s R D R I$ on RNA silencing with $\beta$-glucuronidase or green fluorescent protein DNA/RNA in the mutant lines. The results showed that RNA silencing was impaired in these mutant lines by inverted repeat (IR) DNA or in vitro transcribed double-stranded RNA. Further, the mutant lines were bombarded with Brome mosaic bromovirus (BMV, a ssRNA virus) or Wheat dwarf geminivirus (WDV, a ssDNA virus), each carrying the IR sequence of a reporter gene. As a result, RNA silencing was impaired by BMV. Interestingly, however, it was not impaired by WDV. Thus we propose that OSRDR1 is required for RNA silencing mediated by Bromovirus, but not by Geminivirus in this system.

Keywords Rice RNA-dependent RNA polymerase 1 . RNA silencing - Brome mosaic bromovirus .

Wheat dwarf geminivirus

\section{Introduction}

RNA-dependent RNA polymerases (RDRs) play a fundamental role in various RNA silencing pathways in numerous organisms (Curaba and Chen 2008; Wassenegger and Krczal 2006; Yang et al. 2008). The first $R D R$ was discovered about 40 years ago in a search for enzymes that catalyze plant RNA viral replication (Astier-Manifacier and Cornuet 1971). The first plant-encoded RDR was isolated from tomato (Lycopersicon esculentum), and is now called LeRDRI (Schiebel et al. 1993). In the fungus Neurospora crassa, NcRDR6 (QDE-1) is an essential component of quelling and can convert a single-stranded RNA (ssRNA) to a double-stranded (dsRNA) molecule in a primerdependent/independent fashion (Makeyev and Bamford 2002). In Arabidopsis thaliana, six RDRs were identified as 
AtRDR1, AtRDR2, AtRDR3a, AtRDR3b,AtRDR3c and $A t R D R 6$, three of which (RDR3a, RDR3b, RDR3c) share the unique DFDGD amino acid motif in the catalytic domain (Voinnet 2008; Wassenegger and Krczal 2006). Recent findings indicate that $R D R 1, R D R 2$ and $R D R 6$ act in different biological processes of RNA silencing (Dalmay et al. 2000; Mourrain et al. 2000; Xie et al. 2004). RDRl expression is induced by salicylic acid (SA), a defensesignaling hormone that accumulates during infection by many viruses and viroids (Dorssers et al. 1984; Khan et al. 1986; Xie et al. 2001). More recent studies with Cucumber mosaic virus (CMV) demonstrate that the production of small interfering RNAs (siRNAs) is strongly dependent on $R D R 1$ without interference by the virus-encoded $2 \mathrm{~b}$ silencing suppressor protein called viral suppressors of RNA silencing, and RDRI-dependent secondary virusderived siRNAs (viRNAs) play a key role in non-cellautonomous antiviral silencing (Diaz-Pendon et al. 2007). It also implies that virus encoded proteins may inhibit one or more RDR pathways of siRNA biogenesis associated with silencing suppressor proteins (Donaire et al. 2008), while other reports indicate that $R D R 1$ mediates defense against herbivore attacks in Nicotiana attenuata (Pandey and Baldwin 2007). RDR2 transcripts in $N$. attenuata are up-regulated by both UV and herbivore elicitation for protection (Pandey and Baldwin 2008), while RDR6 is known to be involved in initiation and maintenance of post-transcriptional gene silencing (PTGS) and virus-induced gene silencing (VIGS) (Wassenegger and Krczal 2006). RDR6 is required for VIGS induced by Cabbage leaf curl virus $(\mathrm{CaLCuV})$ and $\mathrm{CMV}$, but not by Turnip crinkle virus (TCV), Tobacco rattle virus (TRV), Turnip mosaic virus or Tobacco mosaic virus (Diaz-Pendon et al. 2007; Muangsan et al. 2004; Vaucheret 2006; Voinnet 2005).

In rice, five $R D R \mathrm{~s}$ have been identified: $O s R D R I$, OsRDR2, OsRDR3a, OsRDR3b and OsRDR6 (Wassenegger and Krczal 2006). A recent study indicates that the OsRDR6-dependent siRNA biogenesis that derives from an isocitrate lyase transcript was significantly up-regulated by the phytohormone abscisic acid (ABA), and OsRDR6 may participate in the amplification of PTGS mediated by ABA in rice (Yang et al. 2008). The function of other $R D R$ enzymes remains a key question. Here we isolated and identified several OSRDR1 mutant lines using the nucleotide sequence of SGS2/SDE1/RDR6 in Arabidopsis as the query to search for the sequences that flank the insertions of rice retrotransposon Tos 17 . We used particle bombardment to establish an RNA silencing system to investigate any effects of OsRDRl on RNA silencing with various silencing inducers, including inverted repeat (IR) DNA or dsRNA, in these mutant lines. We tested the role of OsRDR1 in RNA silencing mediated by Wheat dwarf virus (a ssDNA geminivirus, WDV) or Brome mosaic virus (a ssRNA bromovirus, BMV), each carrying the IR sequence of a reporter gene. Here we used either $\beta$-glucuronidase $(G U S)$ or green fluorescent protein $(G F P)$ to make the data more versatile and to use the viral vectors available, pWI-GUS (Ugaki et al. 1991) and BMV-GFP. Our analyses show that OsRDR1 is required for RNA silencing mediated by BMV, but not by WDV.

\section{Materials and methods}

Rice plants

Oryza sativa L. cv. Nipponbare and its knockout mutant lines (ND2001 and ND2059) were used. The homozygous individuals from M1 and M2 generation were screened by Southern blot analyses. Seedlings and calli of selected M2 plants were grown and used for respective experiments.

\section{Southern blot analysis}

Employing the cetyltrimethyl ammonium bromide (CTAB) method, we isolated rice genomic DNA $(20 \mu \mathrm{g})$ and transferred it to a nylon membrane as described previously (Chen et al. 2008). Using the PCR DIG probe Synthesis Kit (Roche, Basel, Switzerland), the probe was prepared as described previously (Chen et al. 2008). A plasmid carrying the flanking sequences in each mutant was constructed as template DNA in the following protocol: a PCR product amplified from the coding region of $O S R D R l$ by using forward primer ( $5^{\prime}$-AAACCCTTGCCTCCACCCAGGTG- $\left.3^{\prime}\right)$ and reverse primer $\left(5^{\prime}\right.$-ATGGACTATACTCCAGCACC AA $-3^{\prime}$ ), and the fragment amplified was then inserted into pUC19 (Stratagene, La Jolla, CA, USA), which had been digested with SmaI. The probes were labeled according to the manufacturer's instructions. DNA was then fixed to the membrane by UV irradiation, and the prehybridization and hybridization were done as described (Engler-Blum et al. 1993) for subsequent exposure to X-ray film (Boehringer Mannheim, Mannheim, Germany) for 12-18 h. Hybridizations were performed with DIG-dUTP-labeled (digoxigenin-labeled) DNA probes.

DNA sequencing and sequence analysis

DNA sequencing, sequence analysis and homologue searching were carried out as described previously (Chen et al. 2008).

Plasmids used for transient expression

The GFP expression plasmid p35S-GFP and the DsRed (Discosoma red fluorescent protein) expression plasmid 
pUbq-DsRed driven by an enhanced 35S promoter of Cauliflower mosaic virus (CaMV) and an ubiquitin promoter of maize polyubiquitin, respectively, were provided by Miki and Shimamoto (2004). Plasmid GFP RNAi was transcribed into an RNA predicted to form a doublestranded hairpin RNA structure (Miki and Shimamoto 2004). Plasmid pIG121 contains GUS, driven by the CaMV $35 \mathrm{~S}$ promoter and $35 \mathrm{~S}$ terminator, respectively (Ohta et al. 1990). Plasmid pUbq-DsRed was used as an internal control (Miki and Shimamoto 2004). The p35S-GUS RNAi was described previously (Chen et al. 2008).

The plasmid of pWI-GUS is a plant-bacterium shuttle vector derived from WDV having GUS under the control of the $35 \mathrm{~S}$ promoter and terminator (Ugaki et al. 1991). The pWI-GUS RNAi was constructed as follows (Fig. 1a): a fragment containing the GUS sense fragment and $\lambda$ phage sequence was derived from p35S-GUS RNAi through digestion with EcoRI and SacI, cloned to pBluescriptII $\mathrm{KS}+$ (Stratagene), creating $\mathrm{pBS}-\mathrm{GUS}+\lambda$. A PCR product containing a 831-bp linker of $\lambda$ phage and $1300 \mathrm{bp}$ of the inverted-repeat $G U S$ fragment was amplified with pBS-GUS $+\lambda$ as a template using forward primer 5'-GCCGCATGCGAATTCGGACAGACAGTG-3' containing the SphI site (underlined) and reverse primer 5'-GCCGCATGCGGGAATGGTGATTACCGAC-3' containing the SphI site (underlined). The amplified fragment was digested with SphI and inserted into the end of the GUS fragment of pWI-GUS, which had been digested partially with $\operatorname{SphI}\left(5 \mathrm{~min}\right.$ at $\left.37^{\circ} \mathrm{C}\right)$, so that the plasmid pWI-GUS RNAi was constructed after the orientation was selected and verified by sequencing.

The mixture of BMV-GFP or BMV-GFP RNAi (Fig. 1b) was derived from in vitro transcription of pBTF1, pBTF2 and pBTFCP2smGFP or pBTFCP2hpGFP2, respectively (Mori et al. 1993). pBTFCP2smGFP and
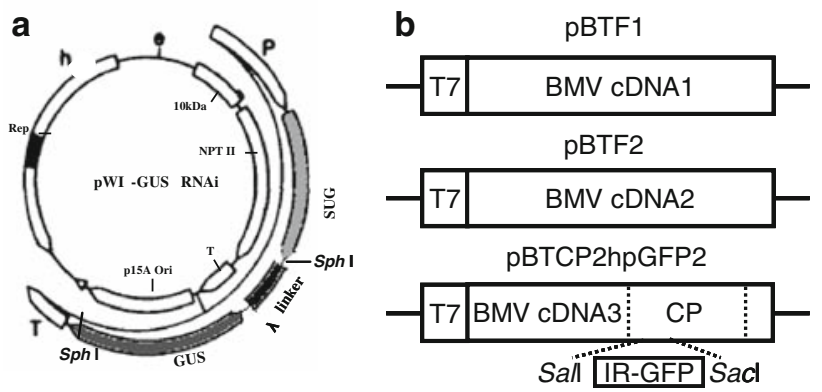

Fig. 1 Diagram of pWI-GUS RNAi (a), which carries IR-GUS; $e$ one of five EcoO109I sites, NPT II neomycin phosphotransferase II genecoding region, $p 15 \mathrm{~A}$ ori replication origin of plasmid p15A, T terminator of CaMV35S transcript, Rep replication-associated protein gene, and BMV RNAi (b), which carries IR-GFP in place of the 5'-half of the CP, $T 7 \mathrm{~T} 7 \mathrm{RNA}$ polymerase promoter
pBTFCP2hpGFP2 were constructed as follows (Fig. 1b): the smGFP gene was amplified by PCR using primers 5'-GGGATGCATGAGTAAAGGAGAAGAACT-3' and 5'-GGGGAGCTCTTATTTGTATAGTTCATCCATGCC-3' from clone smGFP from the Arabidopsis Biological Resource Center at Ohio State University. The PCR product was digested with NsiI and SacI and inserted between the NsiI and SacI site of pBTFCP2pIFN (Mori et al. 1993). The resulting plasmid was digested with NsiI, blunt-ended with T4 DNA polymerase, then self-ligated, creating pBTFCP2smGFP. Then two DNA fragments, the one encoding $0.1 \mathrm{kbp}$ from the C-terminus-, and the other encoding $0.4 \mathrm{kbp}$ from the C-terminus of the GFP gene, was amplified using primer sets \#1554 (5'-ATAACTA GTTTTCGAAAGATCCCAACGAA-3')-\#1553 (5' ${ }^{\prime}$-TATG AGCTCGTCGACTAAAGCTCATCATGTTTGT-3' ${ }^{\prime}$ ), and \#1555 (5'-ATAACTAGTCAAGACACGT-GCTGAAGT CA-3')-\#1553, respectively. As a PCR template, pBI-erG3 (Tamai et al. 2003) was used. The \#1554-\#1553 and the \#1555-\#1553 fragments were digested by SacI/SpeI, and SalI/SpeI, respectively, ligated with SalI/SacI-digested pBTFCP2smGFP. The resulting plasmid was named pBTFCP2hpGFP2, which was engineered to carry the GFP $301 \mathrm{bp}$ as a spacer between an IR of GFP $98 \mathrm{bp}$.

As a complementation study, the p35S-OsRDR1 containing a whole ORF-coding region of cDNA from OsRDRl (3342 bp) was constructed as follows: two RT-PCR fragments (cDNA1, $1769 \mathrm{bp}$ and cDNA2, $1583 \mathrm{bp}$ ) were amplified with forward primer 5'-GGATCCTGCGCCA TGGGTGTCAA- $3^{\prime}$ containing the BamHI site (underlined) and reverse primer 5'-GCATGCTTTTTCTTAAGGAA AG-3' containing the SphI site (underlined) and using

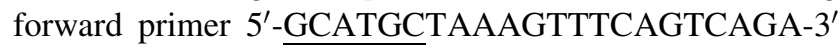
containing the $S p h \mathrm{I}$ site (underlined) and reverse primer 5'-GAGCTCAAACATGCTGGCCACA-3' containing the SacI site (underlined), respectively. The cDNA1 fragment was inserted into the pdTA2 vector (Toyobo, Osaka, Japan). This resulted in pdTA2-cDNA1. The cDNA2 fragment was digested with $S p h \mathrm{I}$ and $S a c \mathrm{I}$ and inserted into pdTA2-cDNA1, which had been digested with SphI and SacI, creating pdTA2-cDNA1 + cDNA2, then this plasmid was digested with BamHI and $\mathrm{SacI}$ to isolate a fragment of cDNA1 + cDNA2, which was gel-purified and inserted into pBI221, which had been digested with BamHI and SacI (to remove GUS). This resulted in p35S-OsRDR1 containing a whole ORF-coding region of cDNA from OsRDRl (3342 bp). The construct was verified by sequencing.

In vitro synthesis of dsRNA

In vitro synthesis of dsRNA for $G U S$ was carried out as described previously (Chen et al. 2008). 
Transient expression after particle bombardment

Gold particles $(0.6 \mu \mathrm{m}$ diameter, particle density $31 \mathrm{mg} / \mathrm{ml}$; Sigma-Aldrich, St. Louis, MO, USA) were coated with the respective mixture of plasmids, then delivered biolistically into leaf segments through a particle inflow gun (IDERA GIE-III, Tanaka, Sapporo, Japan). The samples were bombarded $\left(2.5 \mathrm{~kg} / \mathrm{cm}^{2}\right.$ force and $500 \mathrm{mmHg}$ pressure $)$ with the gold particles coated with the following amounts of plasmids or dsRNA per shot: for RNA silencing with GUS and WDV, (i) pIG121 (5 $\mu \mathrm{g}$ ) and pBI $\Delta$ GUS (control vector) $(5 \mu \mathrm{g})$, (ii) pIG121 (5 $\mu \mathrm{g})$ and $\mathrm{p} 35 \mathrm{~S}-\mathrm{GUS}$ RNAi $(10 \mu \mathrm{g})$, (iii) pIG121 $(5 \mu \mathrm{g})$ and dsGUS RNA $(5 \mu \mathrm{g})$, (iv) p35S-GUS $(5 \mu \mathrm{g})$, p35S-GUS RNAi $(10 \mu \mathrm{g})$ and p35SOsRDR1 $(10 \mu \mathrm{g})$, (v) pIG121 $(5 \mu \mathrm{g})$ and pWI-GUS RNAi $(10 \mu \mathrm{g})$; for RNA silencing with GFP gene and BMV, (i) pUbq-DsRed $(5 \mu \mathrm{g}), \mathrm{p} 35 \mathrm{~S}-\mathrm{GFP}(5 \mu \mathrm{g})$ and pBI221 (control vector) $(5 \mu \mathrm{g})$, (ii) pUbq-DsRed $(5 \mu \mathrm{g})$, p35S-GFP $(5 \mu \mathrm{g})$ and $G F P$ RNAi $(10 \mu \mathrm{g})$; (iii) pUbq-DsRed $(5 \mu \mathrm{g})$, p35S-GFP $(5 \mu \mathrm{g})$, GFP RNAi $(10 \mu \mathrm{g})$ and p35S-OsRDR1 $(10 \mu \mathrm{g})$, (iv) pUbq-DsRed $(5 \mu \mathrm{g})$, p35S-GFP $(5 \mu \mathrm{g})$ and BMV-GFP RNAi $(10 \mu \mathrm{g})$; for BMV replication, pUbqDsRed $(5 \mu \mathrm{g})$ and BMV-GFP $(10 \mu \mathrm{g})$; for WDV replication, pUbq-DsRed $(5 \mu \mathrm{g})$ and pWI-GUS RNAi $(10 \mu \mathrm{g})$. In the case of real-time RT-PCR analysis with GUS silencing, DsRed was used for normalization as a reference gene in all treatments.

After bombardment, the expression of GFP and GUS was detected as described previously (Chen et al. 2008).

\section{RNA isolation and analysis}

Following the manufacturer's instructions (Tri reagent kit, Molecular Research Center, Cincinnati, OH, USA), total RNA was extracted from rice leaves and calli, mRNA was purified using an Oligotex ${ }^{\text {TM }}$-dT30 $<$ Super $>$ mRNA Purification Kit (Takara, Shiga, Japan), and mRNA treated with RNase-free DNase (Stratagene) was used as a template for RT-PCR with reverse transcriptase (MonsterScript $^{\mathrm{TM}}$ 1st-Strand cDNA Synthesis Kit, Epicentre, Madison, WI, USA). RT-PCR was carried out using RT primer $5^{\prime}$-ATACGAGGCGGAATGAGAG-3'. The cDNA products of RT-PCR were then amplified by the following pairs of primers: 5'-GCTTGGATGAAACACGTAC- $3^{\prime}$ (forward) and 5'-ATACGAGGCGGAATGAGAG-3' (reverse). Rice ubiquitin was used as an internal standard (Miki and Shimamoto 2004).

Real-time RT-PCR experiments for detecting the expression of GFP and GUS were carried out as described previously (Chen et al. 2008), and real-time PCR experiments were done with the sets of primers specific to $10 \mathrm{kDa}$ protein of WDV (5'-GGAGGCTTTTGGACCACA TCTTTT- $3^{\prime}$ and $5^{\prime}$-TTCCTCGGTCCTCCTTTGCTTCTT- ${ }^{\prime}{ }^{\prime}$ ).
The amount of DsRed transcripts was used for normalization as an internal control.

\section{Results}

Isolation of homozygous $O s R D R 1$ knockout mutant lines

Homozygous OsRDRI knockout mutant lines from the Tos17 insertion populations available at the Rice Tos 17 Insertion Mutant Database (http://tos.nias.affrc.go.jp/ miyao/ pub/tos17/index.html.en) were selected based on the nucleotide sequence of SGS2/SDE1/RDR6 in Arabidopsis. Two lines (ND2001 and ND2059) had the highest homology to that sequence (referred to as AtRDR6). In these lines, Tos 17 is inserted at the same site as OSRDRl, suggesting that these two were each a callus clone derived from one transformation event because these mutants were selected by tissue culture (Hirochika 2001). The phylogenetic analysis of $O S R D R I$ and other $R D R$ s showed that this gene is most closely related to AtRDRl, and thus it is referred to as OsRDRl (Wassenegger and Krczal 2006). The flanking sequence of each mutant line was used to design the primers and to make the specific DNA probes as shown in the materials and methods. The co-segregation analysis of $\mathrm{X} b a \mathrm{I}$-digested genomic DNA, isolated from each of the individual mutant lines, was carried out by Southern blot analysis; one of three ND2001 and one out of three ND2059 plants were homozygous (Fig. 2a). Furthermore, no significant differences between the wild type (WT) and the homozygous knockout mutant lines have been observed from germination through flowering (data not shown), indicating that the OsRDRl gene is not required for essential biological processes for morphology.

\section{Identification of $O s R D R 1$ homologues in rice}

To identify $O S R D R 1$ in rice, we searched for the SGS2/ $S D E 1 / R D R 6$ sequence in the rice genome sequence database from DNA Bank at the National Institute of Agrobiological Sciences (http://www.dna.affrc.go.jp) and from the chromatin database (http://www.chromdb.org) at the University of Arizona. The flanking sequence of lines ND2001 and ND2059 each had high identity to one genomic clone from chromosome 2, AP004880, one cDNA clone from AK101440 and one transcript sequence from RDR704. Using this information, one putative cDNA of rice $R D R$ was cloned by RT-PCR as described in the materials and methods. Combining these results, AK101440 might be an incomplete clone including a missing transcript in exon 4. Comparison of the putative cDNA and its corresponding genomic sequences revealed that $O s R D R 1$ consists of four 

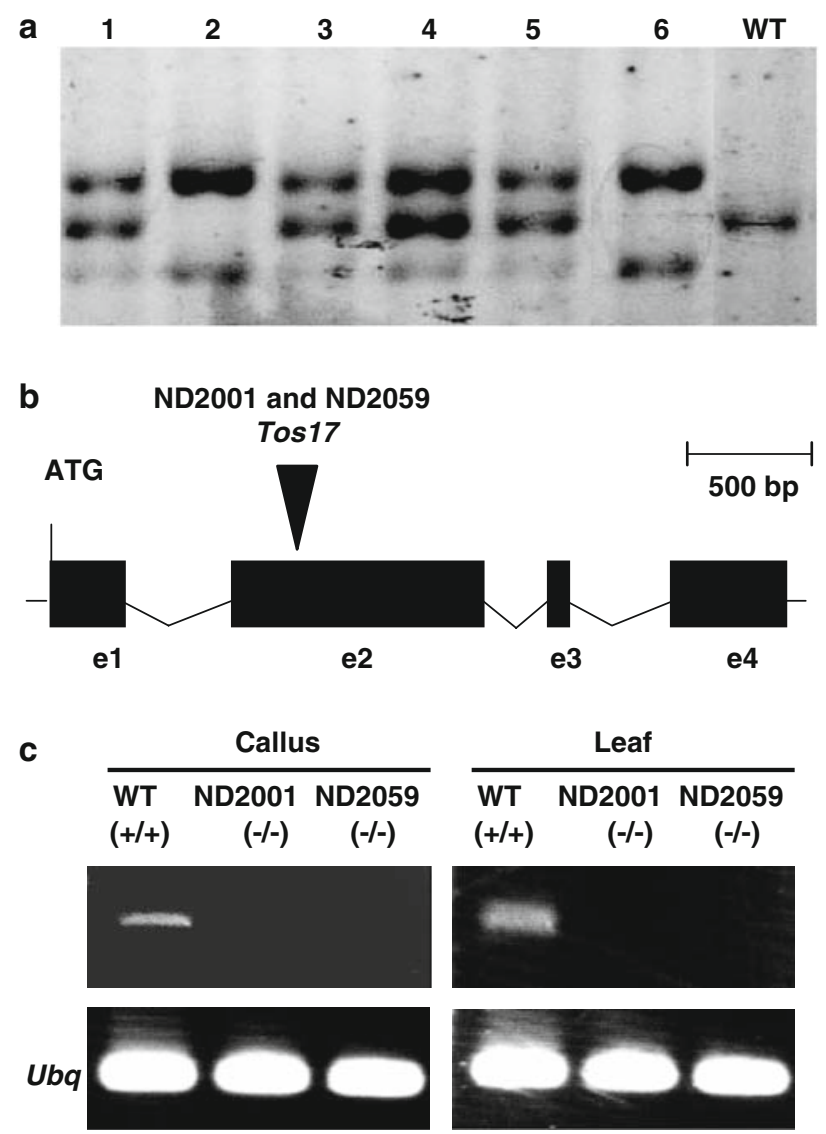

Fig. 2 a Southern blot analysis of $O s R D R 1$ mutant lines. Lanes 1-3 and lanes 4-6 are M1 progeny of lines ND2001 and ND2059, respectively. Lanes 2 and 6 are homozygous in terms of the OsRDRI knockout. b Genomic structure of functional OsRDR1 gene and Tos 17 insertion site in the OsRDR1 knockout mutant lines. Exons (e1-e4) are shown as black boxes. Introns are indicated by broken lines. Black triangles indicate the Tos 17 insertion position in knockout mutant lines. c Comparative RT-PCR of OsRDR1 mRNA from different tissues between wild-type and mutant lines. OsRDR1 mRNA was amplified with 30 PCR cycles. $+/+$ and $-/-$ are the wild-type and mutant line, respectively. Rice ubiquitin $(U b q)$ serves as a normalization control for RT-PCR efficiency

exons interrupted by three introns (Fig. 2b). Two mutant lines have the same Tos 17 insertions in exon 2 (Fig. 2b). A phylogenetic relationship between RDR homologues in rice and Arabidopsis was established. A Neighbor-Joining tree from bootstrap analysis (1000 replicates) was constructed using MEGA software version 3.1. These results indicate that $O s R D R 1$ is in the same clade as $A t R D R I$ with a 99\% consensus value of bootstrap in Fig. S1 in Electronic Supplementary Material.

The expression of OsRDRl in WT and mutant lines (ND2001 and ND2059) was examined with RT-PCR analysis using specific primers for OsRDR1. OsRDR1 mRNA was not detected in the young leaves or calli of the mutants, while a significant level was found in the WT after running 30 PCR cycles (Fig. 2c). These results indicate that $O s R D R I$ was not expressed in the mutant lines due to the insertion of Tos 17 .

Effects of OsRDRI disruption on RNA silencing induced by particle bombardment with IR DNA or dsRNA

As we previously reported (Chen et al. 2008), a transient RNA silencing system targeted to GUS was examined. The different mixtures of plasmids were used. As shown in Fig. 3a, b, substantial levels of $G U S$ were expressed at $48 \mathrm{~h}$ post-bombardment in the histochemical GUS assay of the WT and mutant line ND2001 when bombarded with plasmid (i) pIG121. The number of GUS expressing cells per shot was counted for quantitative analysis in three to six independent experiments ( $n=3-6$ shots). When using mixture (ii) pIG121 and p35S-GUS RNAi, the number of GUS-expressing cells was reduced in the WT but not in the mutant line. When using mixture (iii) pIG121 and dsGUS, a dsRNA for $G U S$ silencing, the number of $G U S$-expressing cells in the WT clearly decreased but not in the mutant line (Fig. 3a, b). The specific silencing of GUS was recovered by co-bombardment with mixture (iv) including plasmid p35S-OsRDR1 in the WT and mutant line (ND2001) (Fig. 3a, b). Moreover, the accumulation of GUS transcripts versus DsRed transcripts (as an internal control) was examined in the leaf discs from the WT and the mutant line at $48 \mathrm{~h}$ post-bombardment with the mixture of those plasmids by real-time RT-PCR (Fig. 3c). Similar results were obtained with three independent experiments essentially as levels of GUS-expressing cells (Fig. 3b). These results were similar to those from the other mutant line (ND2059) (data no shown). Thus, in the mutant lines, RNA silencing was impaired by IR-DNA and dsRNA of GUS.

Effects of $O s R D R 1$ disruption on RNA silencing mediated by plant ssDNA/ssRNA viruses

It is worthwhile examining whether the $O s R D R 1$ gene is required for virus-mediated RNA silencing by particle cobombardment. There are no appropriate viral vectors that infect rice plants available; thus, we selected WDV (ssDNA) and BMV (ssRNA), both of which are reported to infect rice protoplasts (Huntley and Hall 1996; Laufs et al. 1990). Here, experiments with this co-expression system were performed to detect RNA silencing with pIG121 and pWI-GUS RNAi constructed as described in the materials and methods. Specific silencing of GUS was observed after co-bombardment with mixture (v) including plasmid pWIGUS RNAi in the WT and the mutant line (ND2001) (Fig. 3a). This result indicates that $O s R D R I$ is not required for WDV-mediated RNA silencing. To confirm 


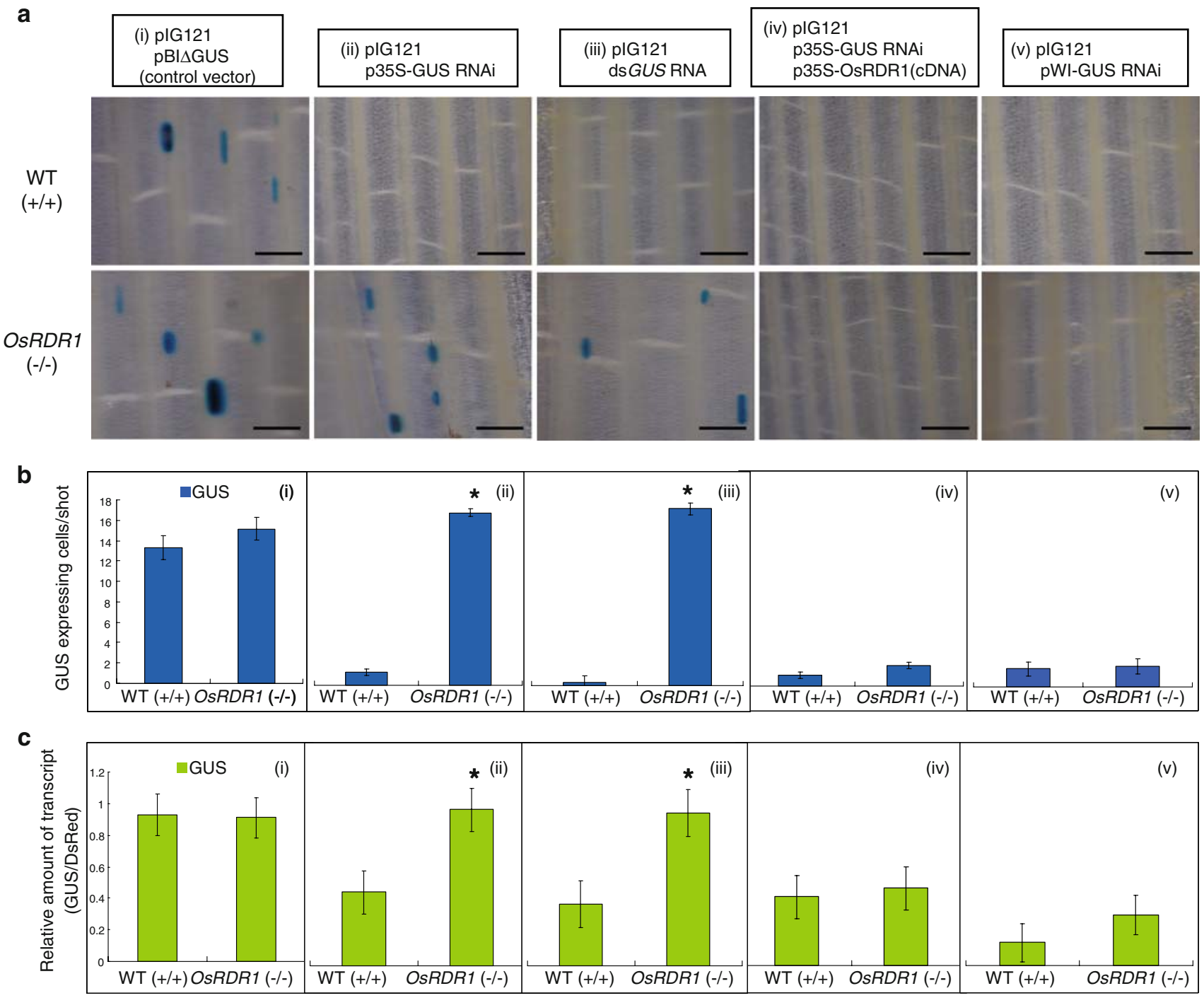

Fig. 3 RNA silencing of GUS in the epidermal cells of rice leaf discs of wild-type (WT) and mutant line after cobombardment with different mixtures of plasmids. a Micrographs of $G U S$ expression. Bar $30 \mu \mathrm{m}$. b Quantitative analysis of GUS-expressing rice cells.

bombardment efficiency, we also examined accumulation of GUS transcripts versus DsRed transcripts (as an internal control) in the leaf discs from the WT and the mutant line at $48 \mathrm{~h}$ post-bombardment with the mixture of those plasmids by real-time RT-PCR (Fig. 3c). These results indicate that the levels of GUS transcript in the leaf discs bombarded were accordant with the levels GUS-expressing cells (Fig. 3a). To check the possibility that WDV interferes with the transcription of $G U S$, an additional experiment has been done with pWI-GUS instead of pIG121. Figure S2a in ESM reveals that both the WT and mutant line (ND2001) expressed GUS (WDV driven) when bombarded with plasmid mixture (i) pWI-GUS and control DNA(pBI $\Delta$ GUS). With mixture (iii) pWI-GUS and pWIGUS RNAi, the number of cells that expressed $G U S$
Data are mean \pm SE ( $n=3-6$ shots). c Real-time RT-PCR analysis of accumulation of GUS transcripts $48 \mathrm{~h}$ after bombardment. Data were normalized using DsRed transcripts as an internal control. Data are mean $\pm \mathrm{SE}(n=3)$. $* P<0.05$ (Fisher's test) versus value of WT

decreased markedly in the WT and in the mutant line, similar to the results shown in Fig. 3a (v). Furthermore, Fig. S2b (i) indicates that DsRed expression remained at a stable level as with GUS (WDV driven) expression. All these results showed that the WDV did not affect expression of GUS or DsRed gene.

We also examined whether BMV affected RNA silencing in the mutant line in this system. For RNA silencing induction, we used BMV-GFP RNAi, in vitro transcripts from BMV cDNA clones. These cDNA clones were the same as reported previously for RNA1 and 2 (Mori et al. 1993). For RNA3, pBTFCP2hpGFP2 was constructed to carry IR-GFP sequence in place of the $5^{\prime}$-half of coat protein gene (CP). When mixture (i) or (ii) in Fig. 4 was used for bombardment, respectively, again 

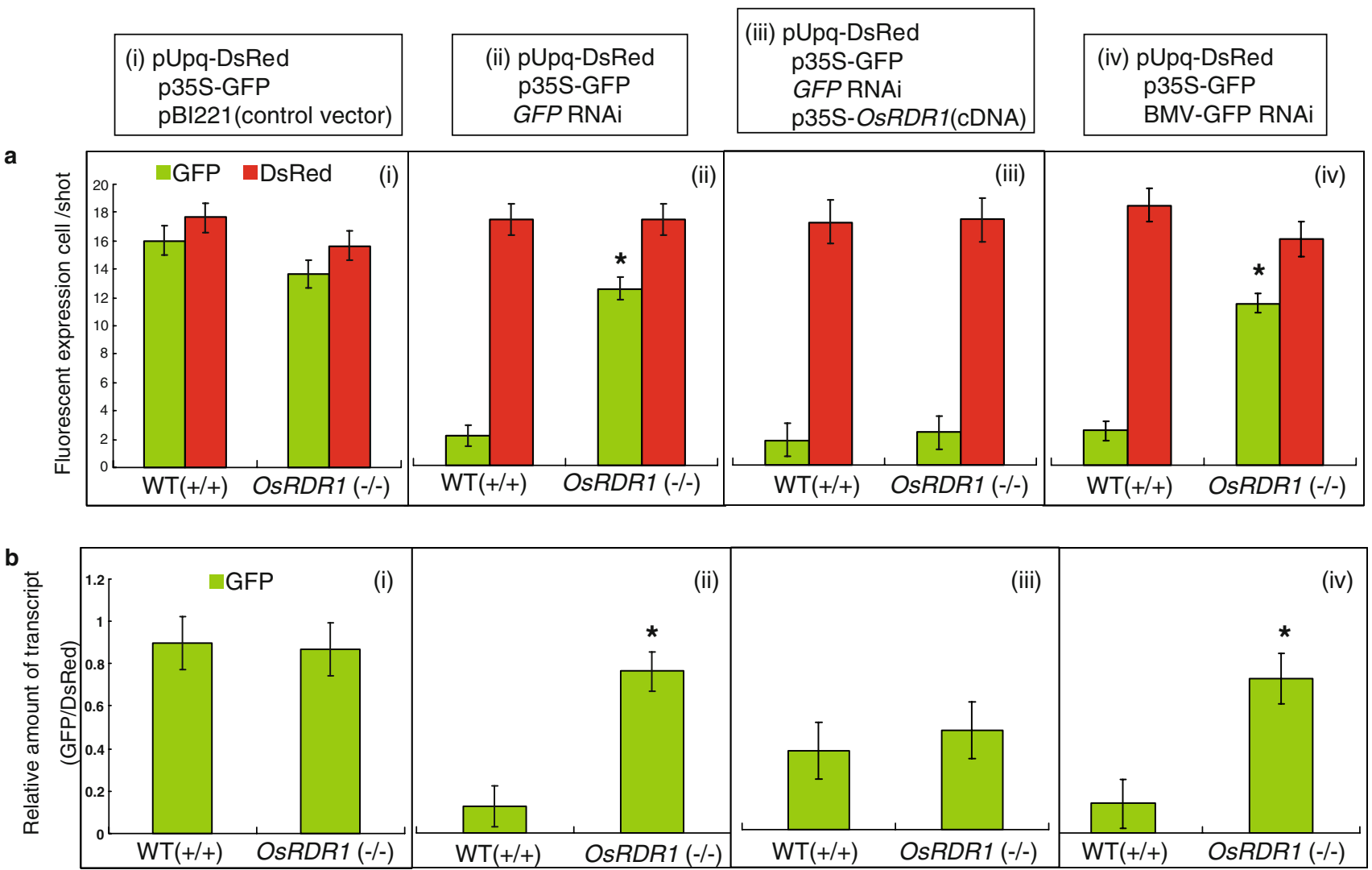

Fig. 4 Effect on RNA silencing mediated by RNA virus in rice leaf discs of wild-type (WT) and mutant line after cobombardment with different mixtures of plasmids. a Quantitative analysis of GFPfluorescing cells per shot. Data are mean $\pm \operatorname{SE}(n=3)$. b Real-time RT-PCR analysis of accumulation of GFP transcripts in the leaf discs

the same results as in Fig. 3 were obtained, showing that RNA silencing of GFP was impaired in the mutant line (Fig. 4a). The number of GFP- or DsRed-fluorescing expressing cells was also counted for quantitative analysis from three independent experiments ( $n=3$ shots). With p35S-OsRDR1 in mixture (iii), which contains a whole ORF-coding region of OsRDRI cDNA, specific silencing of $G F P$ was distinctly recovered in the mutant line, with the number of GFP-fluorescing cells decreasing as markedly in the mutant line as in the WT (Fig. 4a). When using mixture (iv), which contained BMV-GFP RNAi instead of GFP RNAi, there was an obvious decrease in the number of GFP-fluorescing cells in the WT but not in the mutant line, while the number of DsRed-fluorescing cells remained constant in all treatments. To verify bombardment efficiency, we also used real-time RT-PCR to compare the accumulation of GFP transcripts to that of DsRed transcripts in leaf discs from the WT and the mutant line at $36 \mathrm{~h}$ post-bombardment with the various mixtures (Fig. 4b). Similar results were obtained with mutant line ND2059 (data no shown). These results showed that OsRDRl was required for BMV-mediated RNA silencing. at $36 \mathrm{~h}$ after bombardment. Data were normalized using DsRed transcripts as an internal control. Data are mean \pm SE $(n=3)$. The relative amount of transcript $(G F P / D s R e d)$ is shown in the vertical line. $* P<0.05$ (Fisher's test) versus value of WT

To make sure that both viruses are able to replicate in bombarded rice leaf discs, we checked for BMV and WDV replication at different times post-bombardment by realtime RT-PCR and PCR, respectively (Fig. 5). The results show that BMV and WDV were able to replicate in the bombarded leaf discs of both WT and mutant rice.

\section{Discussion}

In fungi, plants and several animals, RDRs are an independent class of enzymes with an important role in RNA silencing, heterochromatin formation and natural gene regulation. Previous findings show that $R D R 6, S D E 3, S G S 3$ and $A G O 1$ are required for sense transgene-mediated PTGS in Arabidopsis, whereas these genes are not involved in IR transgene-mediated PTGS (Dalmay et al. 2000; DiazPendon et al. 2007; Mourrain et al. 2000; Wassenegger and Krczal 2006). In our research, RNA silencing was induced not only by IR-DNAs but also by dsRNA in the WT. However, RNA silencing was impaired by introduction of either IR-DNA or dsRNA in the OsRDRI-deficient mutant 

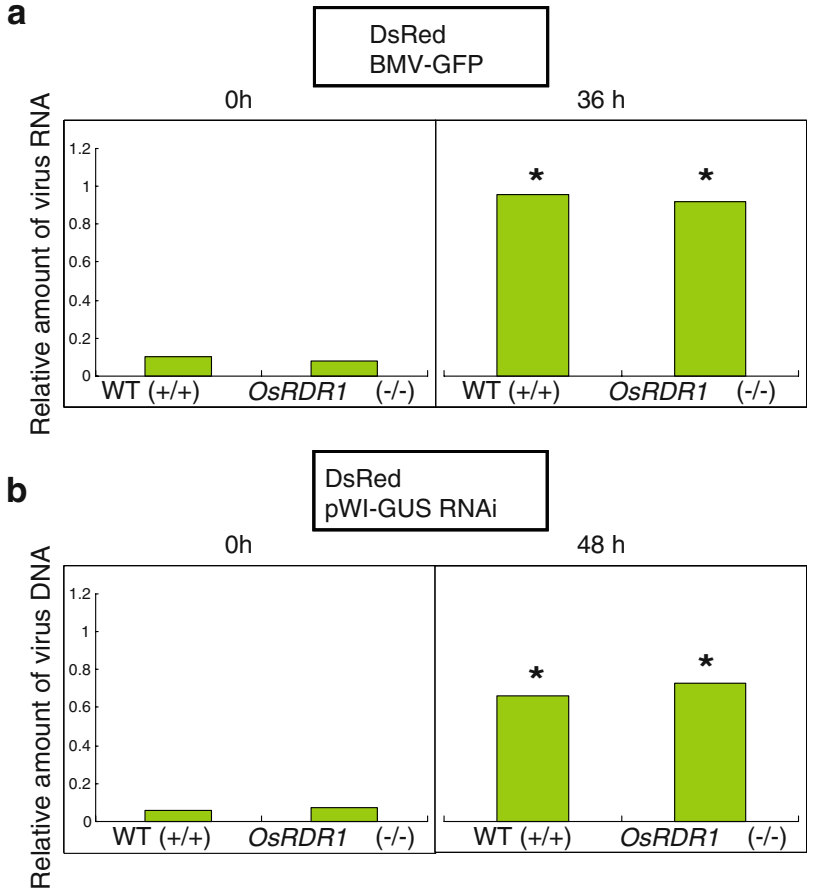

Fig. 5 Analysis of BMV (BMV-GFP) (a) and WDV (pWI-GUS RNAi) (b) replication in bombarded rice leaf discs. Total RNA (a) or DNA (b) was extracted at $36 \mathrm{~h}$ (BMV) or $48 \mathrm{~h}$ (WDV) postbombardment and used as a template for real time RT-PCR or PCR, respectively. Data were normalized using DsRed as an internal control. $* P<0.05$ (Fisher's test) versus value at $0 \mathrm{~h}$

lines. These results suggest that $O S R D R l$ is required at the RNA level after transcription for RNA silencing.

Previous studies show that $R D R I$ is induced by SA treatment, virus and viroid infection (Dorssers et al. 1984; Khan et al. 1986; Wassenegger and Krczal 2006). The lossof-function in the RDRl mutant was expressed as enhanced disease susceptibility to various viruses (Xie et al. 2001; Yu et al. 2003). In Arabidopsis, the accumulation of TRV RNA in the RDR1 mutant was significantly higher than in the WT, and disease symptoms in the mutant appeared to be more severe than in the WT (Yu et al. 2003). In $N$. tabacum, a potato virus $X$ strain could spread and cause a systemic infection in the RDRl mutant line but not in the WT (Xie et al. 2001). Most recently, the finding revealed that the biogenesis of CMV siRNAs was found to be largely dependent on $R D R I$ in plants infected with CMV- $\triangle 2 \mathrm{~b}$, and genes such as SGS3, SDE3 and AGOI might also be essential for $R D R I$-dependent silencing amplification in relation to the production of second siRNAs of CMV (Diaz-Pendon et al. 2007).

In our study, the results from the BMV-GFP RNAi experiments show that $O s R D R 1$ might be attributed to the amplification level of silencing related to the production of second BMV siRNA (Fig. 4a, b). However, WDV-mediated RNA silencing was not impaired in the mutant lines
(Fig. 3a, b), possibly because geminivirus transcription depends on host-DNA-dependent RNA polymerase (Hanley-Bowdoin et al. 2000). VIGS by geminivirus might be amplified by other host $R D R$ s such as RDR2 and RDR6 (Muangsan et al. 2004). Geminivirus DNA was suggested to be methylated by the RNA-directed DNA methylation pathway requiring PolIVa, RDR2, DCL3 and AGO4 in Arabidopsis (Donaire et al. 2008). Thus, WDV in this study is also presumed to be affected in rice by $O s R D R 2$ instead of OsRDRI.

In this RNA-silencing system, we have no data on siRNA, a hallmark of RNA silencing. After co-bombarded with the mixture containing BMV-GFP, GFP RNAi and pUbqDsRed, however, BMV-GFP was clearly reduced (data not shown) like the result shown in Fig. 4, mixture (ii). This finding confirmed that RNA silencing functioned in this system as reported by English et al. (1996). Thus, the siRNA must be produced in each bombarded cell although it is difficult to check at the single cell level. The siRNA could be detected using protoplasts as shown by Qi et al. (2004).

Although rice is not a host for either BMV or WDV (http://www.agls.uidaho.edu/ebi/vdie/sppindex.htm), in some reports, each of these viruses infected and replicated in rice protoplasts (Huntley and Hall 1996; Laufs et al. 1990). Our results in Fig. 5 showed that each virus could at least replicate in the leaf discs after co-bombardment. In the BMV vector used, the $5^{\prime}$-half of CP was replaced by the GFP sequence, which might possibly leave the virus unable to move from cell to cell (Schmitz and Rao 1996). In the WDV vector, the region covering the $\mathrm{CP}$ and part of the movement protein genes was replaced by a selectable marker (NPTII) and GUS (Ugaki et al. 1991). Thus, its cellto-cell movement would be impaired. In Fig. S2a (i) experiment in ESM, only a single cell expressed $G U S$ (data not shown), suggesting that the virus did not spread from cell to cell. Similarly, with BMV vector, GFP was expressed in only one cell (data not shown). The spread GUS/GFP expression after co-bombardment with the wildtype virus would also be worth checking.

Acknowledgments The authors wish to thank K. Shimamoto and D. Miki for plasmids pUbq-DsRed, p35S-GFP and GFP RNAi and K. Nakamura for plasmid pIG121. They are also grateful to D. Murphy for his English correction. This work was supported by the Ministry of Agriculture, Forestry and Fisheries of Japan (Green Technology Project, MP2137). Scholarship for H. C. was supported by the Ministry of Education, Culture, Sports, Science and Technology of Japan.

\section{References}

Astier-Manifacier S, Cornuet P (1971) RNA-dependent RNA polymerase in Chinese cabbage. Biochim Biophys Acta 232:484-493 
Chen H, Samadder PP, Tanaka Y, Ohira T, Okuizumi H, Yamaoka N, Miyao A, Hirochika $\mathrm{H}$, Ohira $\mathrm{T}$, Tsuchimoto $\mathrm{S}$, Ohtsubo $\mathrm{H}$, Nishiguchi M (2008) OsRecQ1, a $Q D E-3$ homologue in rice, is required for RNA silencing induced by particle bombardment for inverted repeat DNA, but not for double-stranded RNA. Plant J 56:274-286

Curaba J, Chen X (2008) Biochemical activities of Arabidopsis RNAdependent RNA polymerase 6. J Biol Chem 283:3059-3066

Dalmay T, Hamilton A, Rudd S, Angell S, Baulcombe DC (2000) An RNA-dependent RNA polymerase gene in Arabidopsis is required for posttranscriptional gene silencing mediated by a transgene but not by a virus. Cell 101:543-553

Diaz-Pendon JA, Li F, Li WX, Ding SW (2007) Suppression of antiviral silencing by cucumber mosaic virus $2 \mathrm{~b}$ protein in Arabidopsis is associated with drastically reduced accumulation of three classes of viral small interfering RNAs. Plant Cell 19:2053-2063

Donaire L, Barajas D, Martínez-García B, Martínez-Priego L, Pagán I, Llave C (2008) Structural and genetic requirements for the biogenesis of Tobacco rattle virus-derived small interfering RNAs. J Virol 82:5167-5177

Dorssers L, van der Krol S, van der Meer J, van Kammen A, Zabel P (1984) Purification of cowpea mosaic virus RNA replication complex: identification of a virus-encoded 110,000-dalton polypeptide responsible for RNA chain elongation. Proc Natl Acad Sci USA 81:1951-1955

Engler-Blum G, Meier M, Frank J, Müller GA (1993) Reduction of background problems in nonradioactive northern and Southern blot analyses enables higher sensitivity than ${ }^{32} \mathrm{P}$-based hybridizations. Anal Biochem 210:235-244

English JJ, Mueller E, Baulcombe DC (1996) Suppression of virus accumulation in transgenic plants exhibiting silencing of nuclear genes. Plant Cell 8:179-188

Hanley-Bowdoin L, Settlage SB, Orozco BM, Nagar S, Robertson D (2000) Geminiviruses: models for plant DNA replication, transcription, and cell cycle regulation. Crit Rev Biochem Mol Biol 35:105-140

Hirochika H (2001) Contribution of the Tos 17 retrotransposon to rice functional genomics. Curr Opin Plant Biol 4:118-122

Huntley CC, Hall TC (1996) Interference with brome mosaic virus replication in transgenic rice. Mol Plant Microbe Interact 9:164170

Khan ZA, Hiriyanna KT, Chavez F, Fraenkel-Conrat H (1986) RNAdirected RNA polymerases from healthy and from virus-infected cucumber. Proc Natl Acad Sci USA 83:2383-2386

Laufs J, Wirtz U, Kammann M, Matzeit V, Schaefer S, Schell J, Czernilofsky AP, Baker B, Gronenborn B (1990) Wheat dwarf virus Ac/Ds vectors: expression and excision of transposable elements introduced into various cereals by a viral replicon. Proc Natl Acad Sci USA 87:7752-7756

Makeyev EV, Bamford DH (2002) Cellular RNA-dependent RNA polymerase involved in posttranscriptional gene silencing has two distinct activity modes. Mol Cell 10:1417-1427

Miki D, Shimamoto K (2004) Simple RNAi vectors for stable and transient suppression of gene function in rice. Plant Cell Physiol 45:490-495

Mori M, Zhang GH, Kaido M, Okuno T, Furusawa I (1993) Efficient production of human gamma interferon in tobacco protoplasts by genetically engineered brome mosaic virus RNAs. J Gen Virol 74:1255-1260
Mourrain P, Béclin C, Elmayan T, Feuerbach F, Godon C, Morel JB, Jouette D, Lacombe AM, Nikic S, Picault N, Rémoué K, Sanial M, Vo TA, Vaucheret H (2000) Arabidopsis SGS2 and SGS3 genes are required for posttranscriptional gene silencing and natural virus resistance. Cell 101:533-542

Muangsan N, Beclin C, Vaucheret H, Robertson D (2004) Geminivirus VIGS of endogenous genes requires SGS2/SDE1 and SGS3 and defines a new branch in the genetic pathway for silencing in plants. Plant J 38:1004-1014

Ohta S, Mita S, Hattori T, Nakamura K (1990) Construction and expression in tobacco of a $\beta$-glucuronidase (GUS) reporter gene containing an intron within the coding sequence. Plant Cell Physiol 31:805-813

Pandey SP, Baldwin IT (2007) RNA-directed RNA polymerase 1 (RdR1) mediates the resistance of Nicotiana attenuata to herbivore attack in nature. Plant J 50:40-53

Pandey SP, Baldwin IT (2008) Silencing RNA-directed RNA polymerase 2 increases the susceptibility of Nicotiana attenuata to UV in the field and in the glasshouse. Plant J 54:845-862

Qi Y, Zhong X, Itaya A, Ding B (2004) Dissecting RNA silencing in protoplasts uncovers novel effects of viral suppressors on the silencing pathway at the cellular level. Nucleic Acids Res 32:e179

Schiebel W, Haas B, Marinković S, Klanner A, Sänger HL (1993) RNA-directed RNA polymerase from tomato leaves. II. Catalytic in vitro properties. J Biol Chem 268:11858-11867

Schmitz I, Rao ALN (1996) Molecular studies on bromovirus capsid protein. I. Characterization of cell-to-cell movement-defective RNA3 variants of brome mosaic virus. Virology 226:281-293

Tamai A, Kubota K, Nagano H, Yoshii M, Ishikawa M, Mise K, Meshi T (2003) Cucumovirus- and bromovirus-encoded movement functions potentiate cell-to-cell movement of tobamo- and potexviruses. Virology 315:56-67

Ugaki M, Ueda T, Timmermans MCP, Vieira J, Elliston KO, Messing J (1991) Replication of a geminivirus derived shuttle vector in maize endosperm cells. Nucleic Acids Res 19:371-377

Vaucheret H (2006) Post-transcriptional small RNA pathways in plants: mechanisms and regulations. Genes Dev 20:759-771

Voinnet O (2005) Induction and suppression of RNA silencing: insights from viral infections. Nat Rev Genet 6:206-220

Voinnet O (2008) Use, tolerance and avoidance of amplified RNA silencing by plants. Trends Plant Sci 13:317-328

Wassenegger M, Krczal G (2006) Nomenclature and functions of RNA-directed RNA polymerases. Trends Plant Sci 11:142-151

Xie Z, Fan B, Chen C, Chen Z (2001) An important role of an inducible RNA-dependent RNA polymerase in plant antiviral defense. Proc Natl Acad Sci USA 98:6516-6521

Xie Z, Johansen LK, Gustafson AM, Kasschau KD, Lellis AD, Zilberman D, Jacobsen SE, Carrington JC (2004) Genetic and functional diversification of small RNA pathways in plants. PLoS Biol 2:e104

Yang JH, Seo HH, Han SJ, Yoon EK, Yang MS, Lee WS (2008) Phytohormone abscisic acid control RNA-dependent RNA polymerase 6 gene expression and post-transcriptional gene silencing in rice cells. Nucleic Acids Res 36:1220-1226

Yu D, Fan B, MacFarlane SA, Chen Z (2003) Analysis of the involvement of an inducible Arabidopsis RNA-dependent RNA polymerase in antiviral defense. Mol Plant Microbe Interact $16: 206-216$ 